\title{
LA CONCURRENCE DES NORMATIVITÉS AU COEUR DE LA LABELLISATION DU BIEN-ÊTRE ANIMAL
}

\author{
Régis BISMUTH \\ Albane DEMARET \\ Alice DI CONCETTO \\ Aude-Solveig EPSTEIN \\ Myele ROUXEL \\ Yann SOUBIGOU 1
}

Revue Internationale de Droit Économique - 2018 - pp. 369-393 - DOI: 10.3917/ride.323.0369

Résumé : Dans un contexte de profonde remise en cause de l'élevage dit « intensif » tendant à englober l'ensemble de la filière élevage sans distinction des pratiques, certains producteurs et partisans d'une meilleure protection des animaux de ferme voient en l'information des consommateurs en matière de bien-être animal un levier d'amélioration du sort des animaux et des acteurs de la filière. Cependant, l'augmentation de la quantité d'information ne se traduit pas toujours par une meilleure transparence quant aux méthodes de production. Au contraire, comme cela est le cas dans les points de vente français, l'excès d'information peut s'avérer aussi délétère que le manque de fiabilité des données communiquées. En l'absence d'une information de qualité, il est à craindre qu'une crise de confiance ne s'installe avant même que l'efficacité d'une régulation par l'information dans le domaine du bien-être animal ait pu être éprouvée. Une analyse des normes en matière de bien-être animal sous le prisme du phénomène de concurrence qui joue entre elles permet de distinguer l'information de qualité de celle de moindre qualité, en vue de prévenir la crise de confiance chez les consommateurs.

1. Les auteurs ont participé durant l'année universitaire 2017-2018 au projet « Labelliser le bien-être animal » au sein de l'École de droit de Sciences Po. Ce projet a été initié par Aude-Solveig Epstein et co-encadré par Régis Bismuth et Alice Di Concetto. Albane Demaret, Myele Rouxel et Yann Soubigou sont étudiants à Sciences Po et ont mené ce projet clinique. Description détaillée du projet disponible en ligne: http://www.sciencespo.fr/ecole-de-droit/fr/content/programme-rise. Régis Bismuth est professeur à l'École de droit de Sciences Po, Alice Di Concetto est chargée de recherche (fellow) au sein du programme de droit de l'animal de l'École de droit de Harvard (Animal Law \& Policy Program at Harvard Law School) et Aude-Solveig Epstein est maître de conférences en droit privé et co-directrice du Collège d'excellence de la Faculté de droit à l'Université de Caen Normandie. 
1 La concurrence entre normes publiques et privées en matière de bien-être animal

1.1 Des normes privées se substituant à des normes publiques insuffisantes :

l'exemple du label néerlandais Beter Leven

1.2 L'émergence de normes privées favorisant l'émergence de la norme publique :

l'exemple de l'étiquetage des œufs

2 L'espace de concurrence des normes du bien-être animal

2.1 La division théorique au sein du champ du bien-être animal : zootechnie et paysannerie

2.2 La géométrie variable de la définition du bien-être animal dans le label

2.3 La géométrie variable du bien-être animal dans la mise en œuvre du label : l'exemple de la concurrence entre les approches empiriques et sensorielles

2.3.1 L'approche empirique

2.3.2 L'approche sensorielle s'appuie sur la relation particulière entre hommes et bêtes

2.3.3 Une approche synergique du bien-être animal : l'exemple de Welfare Quality

De la crise de la vache folle aux vidéos d'abattoirs rendues publiques par l'association L214, les raisons de s'inquiéter des conditions de vie, de transport et d'abattage des animaux d'élevage ne manquent pas, et ce, d'autant plus que la sensibilité et l'intelligence animale s'imposent aujourd'hui comme des réalités scientifiques.

En dépit de l'évolution de l'opinion publique, les partisans de législations plus protectrices des animaux d'élevage continuent à se heurter à des obstacles importants : leurs revendications viendraient saper la liberté de circulation des marchandises, battre en brèche la démocratisation de la consommation de viande animale, mettre en péril l'existence de la filière et avec elle des traditions culinaires, voire culturelles, sans compter qu'elles font l'objet d'une instrumentalisation de la part de groupes xénophobes, à travers la critique de l'abattage rituel.

Dans ce contexte, d'aucuns placent leurs espoirs dans une solution de compromis : l'amélioration de l'information des consommateurs sur la prise en compte du bien-être animal dans le processus de production permettrait l'amélioration croisée du sort des animaux d'élevage et des acteurs de la filière. De fait, si les consommateurs étaient mieux informés sur le sort réservé aux animaux entrant dans la composition des produits qu'ils acquièrent, ils seraient mieux à même de faire converger leurs choix de consommation avec leur préférence éthique pour la protection du bien-être des animaux d'élevage ${ }^{2}$.

Ces dernières années, ce type d'information a de plus en plus proliféré dans les points de vente français. Pour l'heure, il n'existe, il est vrai, aucun label spécifiquement dédié au bien-être animal en France. Cependant, certains labels dits « qualitatifs» incluent dans leur cahier des charges des exigences en termes de

2. Commission européenne, « Attitudes des Européens à l'égard du bien-être animal », Eurobaromètre spécial 442, mars 2016, p. 9. 
bien-être animal (par exemple : Label Rouge, Label Bio, Nature et Progrès). En outre, d'autres formes d'allégations relatives au sort des animaux d'élevage se développent rapidement.

Cependant, l'augmentation de la quantité d'information ne se traduit pas toujours par une meilleure transparence quant aux méthodes de production. $\mathrm{Au}$ contraire, l'excès d'information peut s'avérer aussi délétère que le manque de fiabilité des données communiquées. Or, comme les pouvoirs publics français en ont eux-mêmes fait le constat, l'information donnée aux consommateurs en matière de bien-être animal est de mauvaise qualité, de telle sorte que la " [mise] à disposition des consommateurs [d'] une information objective sur la définition du bien-être animal et sa déclinaison en élevage ${ }^{3}{ }^{3} \mathrm{~s}$ 'impose comme une priorité. En l'absence d'une information de qualité, il est à craindre qu'une crise de confiance ne s'installe avant même que l'efficacité d'une régulation par l'information ${ }^{4}$ dans le domaine du bien-être animal ait pu être éprouvée.

Une analyse des normes en matière de bien-être animal sous le prisme du phénomène de concurrence qui joue entre elles peut permettre dans un premier temps de distinguer l'information de qualité de celle de moindre qualité, en vue de prévenir la crise de confiance chez les consommateurs. Cet article se focalise sur deux espaces de concurrence des normativités. Un premier espace est celui de la concurrence, somme toute assez classique, entre normes publiques et normes privées (1). Le second espace de concurrence des normativités se rapporte aux différentes conceptions du bien-être animal selon des normativités relevant de disciplines et de savoirs différents (biologie, médecine vétérinaire, éthologie, savoir-faire paysan, etc.) qui entrent souvent en concurrence les unes avec les autres pour influencer le contenu des normes publiques et privées relatives au bien-être animal (2). Ces deux espaces de concurrence, entre normes privées et normes publiques d'une part, et entre disciplines sous-tendant le contenu normatif du bien-être animal d'autre part, sont également soumis à des contraintes de type économique.

\section{LA CONCURRENCE ENTRE NORMES PUBLIQUES ET PRIVÉES EN MATIÈRE DE BIEN-ÊTRE ANIMAL}

La prolifération de normes privées en matière d'information du consommateur au sujet du bien-être animal tient notamment au doute existant quant à la possibilité d'imposer un étiquetage obligatoire en matière de bien-être animal au regard $\mathrm{du}$ droit de l'Organisation mondiale du commerce (OMC). En considérant le bien-être

3. Ministère de l'Agriculture et de l'Alimentation, Plan d'action prioritaire en faveur du bienêtre animal, proposition $\mathrm{n}^{\mathrm{o}} 18$, février 2018. Disponible en ligne: http://agriculture.gouv.fr/ 20-actions-prioritaires-en-faveur-du-bien-etre-animal.

4. Sur la régulation par l'information, voy. not. A.-S. EPSTEIN, Information environnementale et entreprise. Contribution à l'analyse juridique d'une régulation, Paris, Institut universitaire Varenne, coll. des Thèses, t. 115, 2015. 
animal comme faisant partie de « processus et méthodes de productions », le droit de l'OMC rend complexe la prise en compte du traitement des animaux ${ }^{5}$.

La prolifération de normes privées en matière de bien-être animal conduit les autorités à manifester des craintes. Par exemple, l'Organisation mondiale de la santé animale (OIE) notait, dans une résolution de 2008, que « les normes commerciales fixées par des sociétés privées sans implication directe des gouvernements interviennent de plus en plus dans le commerce international, et préoccupent la majorité des Membres de l'OIE » et proposait «d'aider les Membres à prendre toutes les mesures applicables pour garantir que les normes privées relatives à la santé et au bien-être des animaux, lorsqu'elles sont utilisées, so[ie] nt cohérentes avec celles publiées par l'OIE et ne so[ie]nt pas en contradiction avec celles-ci $»^{6}$.

Si la préoccupation d'instances publiques, telles que l'OIE, est légitime face à une telle prolifération des normes privées, une labellisation privée peut efficacement suppléer des normes publiques insuffisantes lorsqu'elle offre des gages de sérieux et inclut l'ensemble des parties prenantes (1.1). Dans certaines situations, l'émergence de normes privées peut même servir de base au développement d'une norme publique (2.2).

\subsection{Des normes privées se substituant à des normes publiques insuffisantes : l'exemple du label néerlandais Beter Leven}

Le succès du label néerlandais spécifiquement dédié au bien-être animal Beter Leven (« meilleure vie ») rend compte de l'utilisation de l'information au consommateur comme levier d'amélioration des conditions de vie des animaux d'élevage. Créé en 2007, à l'initiative des militants de la cause animale en réponse à l'inertie du législateur au sujet de l'amélioration des conditions de vie des animaux d'élevage, le label Beter Leven s'est progressivement imposé comme une source d'information au consommateur sur le bien-être des animaux de ferme. L'efficacité de cette démarche témoigne du caractère vertueux des influences réciproques entre normativités publiques et privées, avec dans un premier temps une politique de déréglementation de la part des pouvoirs publics encourageant les initiatives privées, puis l'intégration du label privé à la normalisation publique.

5. De manière générale sur le droit de l'OMC sur cette question, voy. D. Sifonios, Environmental Process and Production Methods (PPMs) in WTO Law, Cham, Springer, 2018.

6. Organisation mondiale de la santé animale (OIE), Implication des normes privées dans le commerce international des animaux et des produits d'origine animale, résolution $\mathrm{n}^{\circ} \mathrm{XXXII} d u$ 29 mai 2008. Sur la concurrence entre normes privées et publiques, voy. aussi R. BismuTH, « Une cartographie de la standardisation internationale privée : tentative d'identification de l'objet et de ses enjeux », in R. Bismuth (dir.), La standardisation internationale privée : aspects juridiques, Bruxelles, Larcier, 2014, pp. 9 et s. 
Jusqu'en 2002, la législation néerlandaise en matière de bien-être animal était la plus exigeante au sein de l'Union européenne (UE), après celle du Royaume-Uni ${ }^{7}$. La loi sur la santé et le bien-être des animaux de 1992 prévoyait notamment une densité de peuplement dans les élevages de porcs supérieure à celle prévue par les directives européennes ${ }^{8}$. Le Gouvernement élu en 2002 ne fit plus de l'amélioration des conditions de vie des animaux de ferme ${ }^{9}$ une priorité, souhaitant au contraire libérer les filières en laissant aux producteurs la liberté de se soumettre à des normes plus contraignantes pour satisfaire la demande des consommateurs ${ }^{10}$. Ainsi, entre 2002 et 2004, le Gouvernement néerlandais adopta une législation relative au bien-être des porcs qui s'alignait à nouveau sur les normes minimales de l'UE tout en incitant les acteurs économiques à adopter des mesures volontairement plus exigeantes. L'émergence et le développement du label Beter Leven ont été favorisés non seulement par ce contexte propice aux initiatives volontaires, mais encore par la crise de confiance des consommateurs suite à une série de scandales sanitaires touchant l'élevage intensif aux Pays-Bas ${ }^{11}$. Dans ce contexte de retrait des pouvoirs publics, la Société protectrice des animaux des Pays-Bas (De Dierenbescherming) entreprit d'occuper l'espace normatif laissé vacant par la déréglementation des normes de bien-être animal par la création en 2007 du label Beter Leven (« meilleure vie »).

Ce label propose trois niveaux d'amélioration du bien-être des animaux. Chaque niveau est identifié par un nombre d'étoiles correspondant aux différentes conditions d'élevage. S'agissant de l'élevage de porcs, le système de notation se décline de la façon suivante :

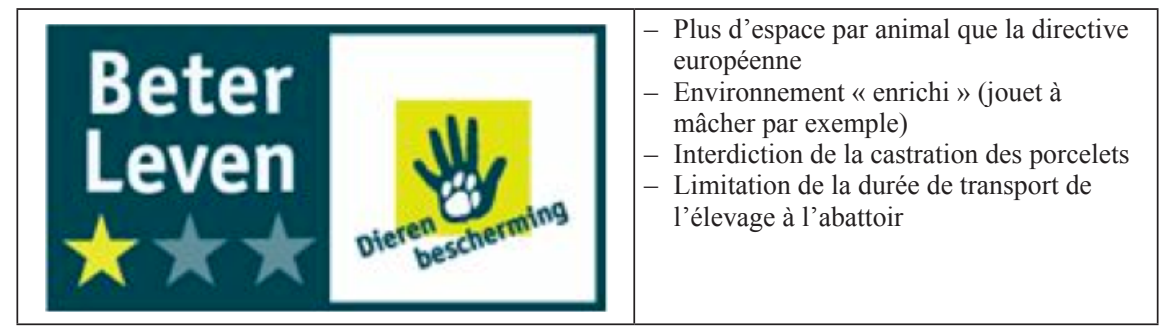

7. B.B. Bock et F. van LeEUwen, « Socio-political and Market Developments of Animal Welfare Schemes », in J. Roex et M. Miele (eds.), Farm Animal Welfare Concerns, Consumers, Retailers and Producers, Welfare Quality Report, Cardiff University, 2005, p. 123.

8. Ibid., p. 119.

9. M.M. van HuIK et B.B. Bock, «Attitudes of Dutch Pig Farmers Towards Animal Welfare », British Food Journal, vol. 109, 2007, n 11, p. 882.

10. Min. LNV, Kiezen voor Landbouw - een visie op de toekomst van de agrarische sector, Ministerie van Landbouw, Natuur en Voedselkwaliteit, 2005 (Min. LNV, Ministère de l'Agriculture, de la Nature et de la qualité de l'Alimentation), Den Haag (en néerlandais).

11. En 2004, par exemple, la mutation d'un agent pathogène transmissible à l'homme en provenance des élevages de porcs avait mis en lumière l'administration excessive d'antibiotiques (à des fins prophylactiques) dans les élevages intensifs, et le risque que cette pratique faisait peser sur la santé publique en participant à l'aggravation du phénomène d'antibiorésistance. Source : Compassion in World Farming, Antibiotics in Animal Farming. Public Health and Animal Welfare, 2011, pp. 18-20. Disponible en ligne : https://www.ciwf.org.uk/media/3758863/Antibiotics-in-AnimalFarming-Public-Health-and-Animal-Welfare.pdf. 


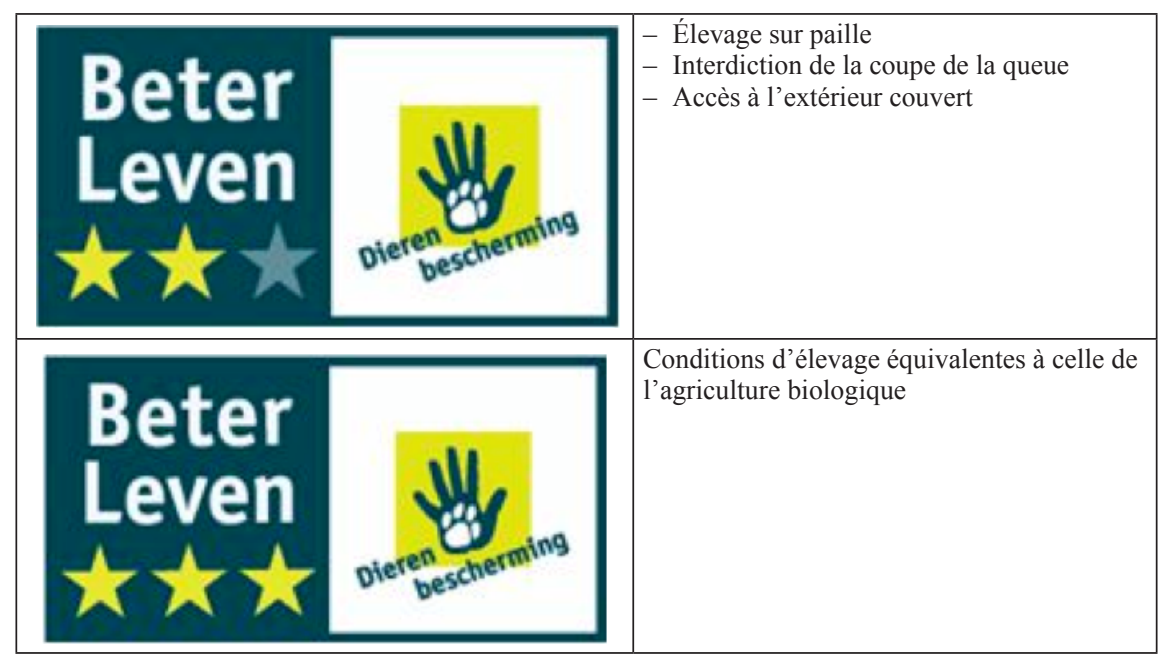

Source : Beter Leven, Over het Kenmerk, 2018. Disponible en ligne : http://beterleven.dierenbescherming.nl (en néerlandais).

En optant pour un label graduel plutôt que pour une certification ne récompensant que les pratiques les plus vertueuses, Beter Leven vise l'intégration d'un maximum de producteurs dans une démarche de progrès. Le label permet également de répondre aux attentes des consommateurs, désormais en meilleure posture pour arbitrer entre leurs contraintes budgétaires et leurs convictions en matière de traitement des animaux d'élevage, et en tout état de cause mieux informés. Au-delà de la démarche de progrès des producteurs, la labellisation Beter Leven vise donc à éclairer les acheteurs par la diffusion de l'information en matière de bien-être animal auprès du consommateur. L'hypothèse de l'information au consommateur comme levier efficace d'amélioration du traitement des animaux dans les chaînes de production alimentaire repose sur l'écart traditionnellement observé entre le souci des citoyens pour le bien-être des animaux d'une part, et les choix des consommateurs d'autre part. Ainsi, aux Pays-Bas, $69 \%$ de la population pense que le bien-être des animaux d'élevage est une question importante, alors que seulement $27 \%$ des consommateurs néerlandais prennent en compte cette question dans leurs décisions d'achat ${ }^{12}$. Ce paradoxe mis en lumière par de nombreuses études ${ }^{13}$ tient notamment à l'insuffisance de l'information du consommateur sur le traitement des animaux lors de la production des biens alimentaires. Dans le cas néerlandais, le manque d'information au consommateur comme facteur explicatif

12. Chiffres datant de 2005. Source: U. KJaernes et R. LaviK, «Farm Animal Welfare and Food Consumption Practices: Results from Surveys in Seven Countries », in U. KJaERnes, M. Miele et J. Roex (eds.), Attitudes of Consumers, Retailers and Producers to Farm Animal Welfare, Welfare Quality Report No. 2, Cardiff University, p. 13.

13. D. Harvey et C. Hubbard, « The Supply Chain's Role in Improving Animal Welfare », Animals, 2013, n 3 , pp. 773-774. 
des comportements de consommation se trouve corrélé par le fait que $85 \%$ des consommateurs néerlandais se disent prêts à payer plus cher pour une production alimentaire plus soucieuse du bien-être des animaux d'élevage, un pourcentage très supérieur à la moyenne européenne, qui est de $59 \%{ }^{14}$. Un tel label qualitatif fondé sur trois niveaux d'exigence au surcoût limité permet ainsi d'atteindre des consommateurs qui auraient choisi des productions standards en l'absence de label ${ }^{15}$.

Le label Beter Leven s'est rapidement généralisé sur le marché néerlandais. Le ministère de l'Agriculture néerlandais rapportait ainsi que $40 \%$ du porc vendu aux Pays-Bas était labellisé Beter Leven en $2013^{16}$, soit six ans seulement après la création du label. L'ampleur de la labellisation Beter Leven aux Pays-Bas tient principalement à l'engagement de chaînes de la grande distribution, telle que Albert Heijn, dont la grande majorité des produits dérivés du porc est labellisée Beter Leven 1 étoile ${ }^{17}$, ou les magasins de discount Aldi, dont $90 \%$ du porc frais vendu est également labellisé Beter Leven ${ }^{18}$. Si la production porcine illustre particulièrement bien le succès du label Beter Leven, les Pays-Bas étant un des principaux pays producteurs en Europe, ce label a également été adopté par des producteurs de poules pondeuses, de poulets, de bovins, de veaux, de dindes et de lapins. Selon De Dierenbescherming, le programme a permis l'amélioration du bien-être de plus de 100 millions animaux d'élevage ${ }^{19}$.

Malgré cette généralisation, le succès du label Beter Leven dans l'amélioration des conditions d'élevage des animaux demeure mitigé dans la mesure où la majorité des produits labellisés ne le sont qu'au niveau d'entrée « 1 étoile ». En outre, de nombreux produits labellisés « 2 étoiles » disponibles dans les magasins Albert Heijn sont importés d'Angleterre ou d'Écosse ${ }^{20}$.

Si les succès du label Beter Leven demeurent limités au regard de l'évolution des pratiques au sein de l'industrie des productions animales, Beter Leven est néanmoins parvenu à convaincre les pouvoirs publics de s'intéresser à nouveau à la question du bien-être animal par le biais de la commande publique. Ainsi, le ministère de l'Infrastructure et de l'Environnement se réfère désormais aux exigences du label parmi les critères de sélection de fournisseurs de la commande

14. Commission européenne, «Attitudes des Européens à l'égard du bien-être animal », op. cit., p. 15.

15. L.R. Heerwagen, M.R. Mørkbak, S. Denver, P. Sandøe et T. Christensen, « The Role of Quality Labels in Market-driven Animal Welfare », Journal of Agricultural and Environmental Ethics, vol. $28,2015, \mathrm{n}^{\circ} 1$, p. 81 .

16. Ministerie van Ekonomische Zaken, Monitor Duurzaam Voedsel 2012, 2013, http://edepot.wur.nl/ 260072 (en néerlandais).

17. A. HeIJn, Dierenwelzijn/Varken. Check de herkomst, https://www.ah.nl/over-ah/meer-doen/dierenwelzijn/varken.

18. https://www.varkensinnood.nl/nieuwsartikelen/aldi-heeft-hoogste-percentage-beter-levenkeurmerk-bij-het-verse-varkensvlees.

19. Dierenbescherming (n.d.), Boeren Bedankt, https://beterleven.dierenbescherming.nl/beter-boeren/ boeren-bedankt (en néerlandais).

20. C'est le cas de la totalité des produits transformés à base de porc. A. Heisn, Dierenwelzijn/Varken. Check de herkomst, op. cit. 
publique $^{21}$. Selon ces critères, tout fournisseur souhaitant conclure un contrat avec l'État doit a minima respecter le niveau « 1 étoile » du label Beter Leven, qui exige des mesures plus contraignantes en matière de bien-être animal que celles autorisées en droit. En faisant référence à la démarche Beter Leven, la commande publique, une normativité publique de nature contractuelle, a contribué à légitimer le label tout en renforçant sa généralisation sur le marché néerlandais.

De ce point de vue, le label Beter Leven illustre les synergies possibles entre normes publiques et privées. D'un côté, les pouvoirs publics néerlandais ont largement concouru à la légitimation et à l'institutionnalisation du label Beter Leven, pérennisant ainsi sa présence dans le paysage du secteur alimentaire néerlandais. D'un autre côté, le succès du label Beter Leven a donné un nouveau souffle aux initiatives publiques relatives au bien-être animal.

\subsection{L'émergence de normes privées favorisant l'émergence de la norme publique : l'exemple de l'étiquetage des œufs}

Le développement de l'étiquetage des œufs dans l'Union européenne prend ses racines dans une initiative commerciale privée s'apparentant alors à une démarche de labellisation, laquelle poussa les pouvoirs publics à intervenir pour réglementer l'étiquetage des œufs selon le mode d'élevage. Cette intervention se fit en plusieurs temps, qui marquèrent chacun des réactions différentes de la norme publique à la norme privée. La norme publique a été dans un premier temps adoptée en réaction contre une norme privée contestataire dont elle prenait le contrepied au nom de l'information du consommateur et de l'efficacité du marché. Dans un deuxième temps, la norme publique s'est approprié la méthode préconisée par la norme privée sans toutefois en relayer les objectifs, en indiquant certes le mode d'élevage sur le produit, mais en affaiblissant la portée d'une telle information sur le choix des consommateurs. Ce n'est que dans un troisième temps que la norme publique a repris dans son entièreté le sens premier de la démarche de la norme privée en rendant impérative la mention du mode d'élevage.

Dans les années 1960, l'éleveur français Pierre Rannou entendit différencier les œufs de production au sol des œufs issus de l'élevage de poules pondeuses en batterie en apposant l'allégation "Garantie de poules libres en poulaillers clairs Soleil - Air pur $»^{22}$ sur ses boîtes. Or cette mention contrevenait au règlement communautaire de 1975, par lequel la Communauté économique européenne fixait les normes de commercialisation applicables aux œufs ${ }^{23}$. L'ambition première de cette

21. Ministère de l'Infrastructure et de l'Environnement, Criteria for Sustainable Public Procurement of Catering, novembre 2012, https://english.rvo.nl/sites/default/files/2013/12/Criteriadocument $\% 20$ Catering.pdf.

22. Fondation Droit animal, éthique et sciences, «L'étiquetage des boîtes d'œufs », http://www. fondation-droit-animal.org/impacts/etiquetage-boites-oeufs/.

23. Règlement (CEE) $n^{\circ} 2772 / 75$ du Conseil du 29 octobre 1975 concernant certaines normes de commercialisation applicables aux œufs. 
législation était l'amélioration de l'information du consommateur tout en évitant les obstacles techniques liés aux normes de commercialisation des œufs sur le marché unique. Si le règlement permettait effectivement l'apposition d'indications sur les œufs et emballages, il limitait strictement cette possibilité à l'indication de la qualité et du poids des œufs, excluant de ce fait la délivrance d'une information sur le mode d'élevage ${ }^{24}$. Une telle restriction répondait alors à un objectif de rationalisation de l'information disponible au consommateur et de lutte contre la prolifération d'allégations et autres initiatives privées ${ }^{25}$. L'application du règlement aboutit à l'injonction faite à Rannou de retirer la mention non conforme de ses produits, celle-ci ne rendant compte ni de la qualité ni du poids des œufs commercialisés, provoquant la faillite de l'éleveur.

Suite à « l'affaire Rannou », les partisans d'un étiquetage selon le mode d'élevage s'appuyèrent sur la motivation première de l'adoption du règlement de 1975, l'information du consommateur, pour obtenir un changement de législation. Les associations de défense du bien-être animal et de défense des consommateurs se structurèrent en une coalition en 1981, la Coalition des consommateurs contre l'élevage en batterie ${ }^{26}$, et avancèrent des arguments ayant trait à la protection de la santé publique, évoquant notamment le faible apport nutritif des œufs issus d'élevages en batterie et la présence de résidus médicamenteux des œufs produits en élevages intensifs ${ }^{27}$. En parallèle, la coalition intenta également des actions en publicité mensongère contre les producteurs dont les allégations induisaient les consommateurs à penser que les œufs étaient issus d'un mode d'élevage fermier au sol, alors qu'ils provenaient en réalité d'une production en batterie ${ }^{28}$. Au terme de dix ans d'actions militantes - par voie de justice, lobbying et campagnes auprès du public - les efforts de la coalition portèrent enfin leurs fruits : en 1984, un nouveau règlement communautaire introduisit la possibilité d'apposer sur les emballages des « indications destinées à la promotion des ventes ${ }^{29}$, dont le mode d'élevage.

Soucieuses du fait que l'information du consommateur puisse conduire à entraver la libre circulation des biens au sein de l'espace communautaire, les autorités publiques ne renoncèrent pas pour autant à encadrer cette norme de commercialisation sur les emballages. Tout d'abord, l'apposition du mode d'élevage sur l'emballage des œufs demeurait facultative. Ensuite, le règlement de 1984, au motif de garantir la délivrance d'une information claire au consommateur, définissait une liste exhaustive des mentions autorisées portant sur le mode d'élevage des poules pondeuses. Ainsi, seules les mentions « œufs de poules élevées en plein

24. Ibid., considérant 2 et article 21.

25. Ibid., considérant 7.

26. D. Ricaud, «Avant les œufs il y a la poule ! », Revue droit animal, éthique et sciences, 2017, $\mathrm{n}^{\circ}$ 94, pp. 10-12.

27. B. Sauveur, A. Zybko, B. Colas, M. Garreau et J. Rocard, « Protéines alimentaires et qualité de l'œuf », Annales de zootechnie, vol. 28, 1979, n³, pp. 271-295.

28. D. Ricaud, « Avant les œufs il y a la poule ! », op. cit.

29. Règlement $n^{\circ} 1831 / 84$ du 19 juin 1984 modifiant le règlement $n^{\circ} 2772 / 75$ concernant certaines normes de commercialisation applicables aux œufs, article 1(5)(c). 
air système extensif », «œufs de poules élevées en plein air », "œufs de poules élevées au sol », "œufs de poules élevées en volière » étaient autorisées ${ }^{30}$. Les œufs provenant d'élevages en batterie n'ayant pas de mention consacrée, seule l'absence de mention sur l'emballage des œufs indiquait la provenance d'élevage intensif. Ainsi, bien que le règlement de 1984 marquât une prise en compte des modes d'élevage dans la norme publique, le texte exprimait également la volonté des pouvoirs publics de garder le contrôle de l'information afin de limiter les effets de l'étiquetage du mode d'élevage sur le choix du consommateur dans le but affiché de préserver le bon fonctionnement du marché commun, mais aussi de protéger les intérêts des filières agroalimentaires.

Le règlement de $2008^{31}$, aujourd'hui en vigueur, donne toute sa force à une norme publique sur la commercialisation des œufs en faisant de la mention du mode d'élevage sur les emballages une obligation pour la commercialisation de tous les œufs sur le marché européen ${ }^{32}$. De plus, ce règlement complète la liste des mentions, désormais obligatoires, par la mention "œufs de poules élevées en cage » pour les œufs issus d'élevages en batterie. Cette obligation légale repose sur la définition réglementaire des modes d'élevage de la directive de $1999^{33}$ établissant les normes minimales relatives à la protection des poules pondeuses, texte empreint d'une volonté de faire du bien-être animal un objectif des politiques publiques. Par cette directive, le règlement de 2008 rejoint la vision de Rannou : l'information du consommateur sur le niveau de bien-être animal d'un produit comme outil de différenciation sur un marché concurrentiel.

L'évolution de la réglementation des normes de commercialisation des œufs ainsi retracée illustre ainsi que les normes privées peuvent précipiter le développement de la normativité publique.

\section{L'ESPACE DE CONCURRENCE DES NORMES DU BIEN-ÊTRE ANIMAL}

Au-delà de la concurrence entre normativités publiques et privées, des normativités non juridiques s'affrontent en matière de bien-être animal. À la multiplicité de labels privés relatifs au bien-être animal correspond une multiplicité d'approches scientifiques du bien-être animal, qui est défini et mis en œuvre de façon distincte

30. Règlement CEE $n^{\circ} 1020 / 85$ du 12 juillet 1984 modifiant le règlement CEE $n^{\circ}$ 95/69 en ce qui concerne certaines normes de commercialisation applicables aux œufs, article 11 (7).

31. Règlement $\mathrm{CE} \mathrm{n}^{\circ} 589 / 2008$ du 23 juin 2008 portant modalités d'application du règlement $\mathrm{CE}$ $\mathrm{n}^{\circ} 1234 / 2007$ du Conseil en ce qui concerne les normes de commercialisation applicables aux œufs.

32. Ibid., article $11:$ : les emballages contenant des œufs de catégorie A portent sur la face extérieure de manière facilement visible et parfaitement lisible une indication du mode d'élevage ». Voy. aussi les articles 16, 21, 30 du même règlement.

33. Directive 1999/74/CE du Conseil du 19 juillet 1999 établissant les normes minimales relatives à la protection des poules pondeuses. 
dans chaque label. Les acteurs définissent leurs conceptions du bien-être animal en fonction de leurs objectifs et des contraintes auxquelles ils sont exposés, ce qui explique dans une large mesure la grande variabilité du périmètre de ce que constitue le bien-être animal en fonction de ces diverses perspectives. Diverses normativités du bien-être animal entrent ainsi en concurrence, faisant appel à des disciplines scientifiques diverses (biologie, neurobiologie, médecine vétérinaire, éthologie, sciences cognitives, etc.) d'une part, et à un savoir-faire plus ancien, mais moins normé : la paysannerie. Cette concurrence fait apparaître une forme de « self service normatif $»^{34}$ lorsque les différents acteurs s'emploient à définir leur conception du bien-être animal. C'est dans ce cadre qu'il convient de souligner que le bien-être animal demeure un champ d'étude fragmenté et divisé (2.1). Les différentes définitions du bien-être animal selon les labels (2.2) et l'évaluation du bien-être animal en tant que « délivrable » au stade de la mise en œuvre du label (2.3) illustrent cette concurrence entre différentes normativités scientifiques et savoir-faire paysan.

\subsection{La division théorique au sein du champ du bien-être animal : zootechnie et paysannerie}

Le champ du bien-être animal est traversé par un problème épistémologique tenant aux représentations culturelles et éthiques très variables des différents acteurs qui le composent ${ }^{35}$. Dans le champ d'étude du bien-être animal, un courant envisage le bien-être des animaux comme un phénomène scientifique mesurable de manière objective par des indicateurs. Un autre courant critique la notion même de bienêtre animal, à l'origine développée par l'élevage dit « intensif », recouvrant des mesures technico-managériales ayant accompagné l'industrialisation des procédés de production. Ce courant envisage plutôt le bien-être animal comme la prise en compte du caractère vivant de l'animal par l'homme dans la relation qu'ils nouent, et d'une facette du plaisir que les hommes et les animaux tirent de cette relation.

L'émergence de la problématique du «bien-être animal » va en effet de pair avec l'industrialisation de l'élevage dans les années 1970-1980 en France, par la rapide inclusion dans la zootechnie (la science de l'exploitation de l'animal) de normes avant tout relatives à la santé physique et mentale des animaux de rente ${ }^{36}$. Le bien-être animal a toujours constitué une composante de la logique productiviste de l'élevage intensif, notamment pour satisfaire la «demande sociale ${ }^{37}$

34. V. A. Supıт, « Du nouveau au self-service normatif : la responsabilité sociale des entreprises », in Analyse juridique et valeurs en droit social. Études offertes à Jean Pélissier, Paris, Dalloz, 2004, pp. 541-558.

35. D. Fraser, Understanding Animal Welfare: The Science in its Cultural Context, Oxford-Ames, Wiley-Blackwell, 2008, pp. 241 et s.

36. J. Porcher, «Défendre l'élevage, un choix politique », Contretemps. Revue de critique communiste, https://www.contretemps.eu/defendre-lelevage-un-choix-politique/.

37. Les politiques publiques européennes en matière de bien-être animal considèrent la « demande sociétale » comme le fondement de l'intervention des instances européennes, révélant ainsi une 
persistante de bien-être des animaux d'élevage ${ }^{38}$. Mais loin de remettre en cause les dogmes productivistes de l'industrialisation de l'élevage, les normes élaborées pour rendre compte du bien-être des animaux en élevage tendent à opérer la confusion entre bien-être et santé animale ${ }^{39}$. Ce faisant, ces normes se construisent davantage dans une optique d'adaptation de l'animal aux conditions industrielles que d'amélioration de leur traitement par une réforme des méthodes de production des animaux en tant que biens de consommation de masse. Les travaux de Temple Grandin, zootechnicienne américaine spécialiste du bien-être animal, illustrent le mieux cette perspective du bien-être animal comme technique de management de l'élevage intensif. L'amélioration des parcours à l'entrée des abattoirs industriels, évitant aux animaux de se blesser, d'assister à la mort de leurs congénères et donc permettant de maintenir les niveaux de stress des animaux au plus bas ${ }^{40}$, ou encore la mécanisation des processus d'abattage rituel par la généralisation de «box » d'abattage ${ }^{41}$ sont autant de pratiques devenues caractéristiques de l'élevage industriel, emblématiques d'une conception technique, de type médical, du bien-être animal au service de la rationalité économique ${ }^{42}$.

Les tenants d'une vision zootechnique du bien-être animal sont eux-mêmes en divergence selon qu'ils définissent plus ou moins restrictivement la composante du bien-être animal dans les modèles intensifs. Si David Fraser fait ainsi l'effort d'inclure dans la définition du bien-être animal une composante relative aux conditions naturelles de vie des animaux, une telle conceptualisation se limite néanmoins à définir le bien-être de l'animal en creux par une absence de souffrance physique et de frustration émotionnelle, rendue possible par l'expression de « comportements naturels » prédéterminés par des « impératifs biologiques de l'espèce $»^{43}$. Cette vision technico-managériale du bien-être animal correspond à celle des « Cinq Libertés » ${ }^{44}$ qui sous-tend la législation européenne, imposant par exemple la mise en place de cages dites « aménagées » dans les élevages hors-sol.

vision de la politique réduite à un marché au sein duquel sont administrées les demandes des consommateurs-citoyens. C'est notamment le cas de la proposition de réforme de la Politique agricole de la Commission européenne de juin 2018, classifiant le bien-être animal dans la catégorie des demandes sociétales, article 6(1)(i).

38. J. Porcher, « L'esprit du don : archaïsme ou modernité de l'élevage ? Éléments pour une réflexion sur la place des animaux d'élevage dans le lien social », Revue du MAUSS 2, 2002, nº 20, p. 247.

39. Confusion qui tend à persister encore aujourd'hui, en témoigne le mandat normatif assuré par l'OIE en matière de bien-être animal.

40. T. Grandin, Design of Chutes, Ramps, and Races for Cattle, Pigs, and Sheep at Slaughter Plants, http://www.grandin.com/design/chute.ramp.race.design.html.

41. T. Grandin, ASPCA Pen, 2010, http://www.grandin.com/ritual/rec.ritual.slaughter.html.

42. J. Porcher, « Défendre l'élevage, un choix politique », op. cit.

43. D. Fraser, Understanding Animal Welfare: The Science in its Cultural Context, op. cit., p. 230.

44. Le modèle des « Cinq Libertés » a été théorisé dans un rapport sur le bien-être des animaux dans les élevages intensifs de Roger Brambell en 1965. Commissionné par le gouvernement britannique, le rapport préconisait d'assurer cinq libertés aux animaux : celle de ne pas souffrir de la faim ou de la soif; de ne pas souffrir d'inconfort; de ne pas souffrir de douleurs, de blessures ou de maladies; de pouvoir exprimer les comportements naturels propres à l'espèce ; de ne pas éprouver de peur ou de détresse. Ces cinq principes constituent le socle de toute norme relative au bien-être animal et sont notamment repris dans la définition que donne l'OIE du bien-être animal. 
Ainsi, l'installation de pans de plastique dans les cages de poules pondeuses leur permettrait de se dissimuler lors de la ponte, et donc de réduire les niveaux de stress des animaux par la simple simulation d'un comportement naturel. De même, la mise à disposition d'objets à mastiquer dans les élevages de porcs répondrait à des besoins biologiques, tout en réduisant à la marge les cas de caudophagie qui caractérisent la production porcine.

À rebours de cette conception du bien-être animal comme champ technique dérivé de l'industrialisation de l'élevage, le répertoire des pratiques de la paysannerie envisage le bien-être animal comme un plaisir de vivre partagé entre l'animal et l'éleveur ${ }^{45}$. Cette seconde conception du bien-être animal, qui s'enracine dans un savoir-faire plurimillénaire, prend pleinement en compte le lien existant entre l'homme et l'animal dans le cadre de l'activité qui les unit: l'élevage ${ }^{46}$. Moins théorisée que la première, cette conception du bien-être animal en paysannerie transparaît avant tout au travers de témoignages d'éleveurs sur le lien qu'ils entretiennent avec leurs bêtes ${ }^{47}$. Contrairement au calcul utilitariste de la discipline zootechnique, une telle conception du bien-être animal implique de repenser l'élevage comme une activité n'ayant pas pour seule finalité la production de matière animale destinée à la consommation, mais une activité plurielle où le plaisir que prennent les animaux domestiques et les éleveurs à vivre ensemble a au moins autant d'importance que le revenu que les éleveurs tirent de leur mort ${ }^{48}$. Cette prise en compte globale de la « bonne vie » de l'animal a récemment été remise au goût du jour par le concept de « sentience ${ }^{49}$, qui ne renvoie pas seulement à la faculté de l'animal à ressentir la douleur ou les plaisirs physiques, mais considère l'animal comme un être capable d'éprouver du plaisir à exister. Le clivage entre les deux approches du bien-être animal se trouve magnifié en aval, dans la mise en application des critères évaluant le bien-être animal dans le processus de labellisation.

\subsection{La géométrie variable de la définition du bien-être animal dans le label}

Le clivage présent dans la théorie du champ du bien-être animal se trouve magnifié lors de la mise en application de ce que serait le bien-être animal au sein des labels.

45. J. Porcher, « Le "bien-être animal" existe-t-il ? », Économie rurale, n 285, 2005, p. 7.

46. Voy. généralement, J. PorCher, « Défendre l’élevage, un choix politique », op. cit.

47. J. Porcher, « L'esprit du don : archaïsme ou modernité de l'élevage ? Éléments pour une réflexion sur la place des animaux d'élevage dans le lien social », op. cit., pp. 250-251 ; B.E. Rollin, « The End of Animal Husbandry », in D. Iмноғ, CAFO: The Tragedy of Industrial Farm Factories, University of California Press, 2010.

48. J. PorCHER, « L'esprit du don : archaïsme ou modernité de l'élevage ? Éléments pour une réflexion sur la place des animaux d'élevage dans le lien social », op. cit., p. 250, note 15 et généralement J. Porcher, Vivre avec les animaux, une utopie pour le XXI' siècle, Paris, La Découverte, 2011.

49. Pour une définition de la «sentience», voy. A. Guillaume, "Sentience" : reconnaissons la conscience et la sensibilité des animaux », Marianne, 10 octobre 2017, https://www.marianne. net/debattons/idees/sentience-reconnaissons-la-conscience-et-la-sensibilite-des-animaux. 
L'analyse comparée ${ }^{50}$ des cahiers des charges de ces labels européens ou américains $^{51}$ et des réglementations relatives aux labels publics ${ }^{52}$ conduit tout d'abord à constater l'absence de définition claire du bien-être animal, reflétant l'absence de consensus sur la notion de bien-être animal. Quatre catégories de définitions du bien-être animal se dégagent néanmoins de l'analyse des cahiers des charges des labels portant sur le bien-être animal ${ }^{53}$.

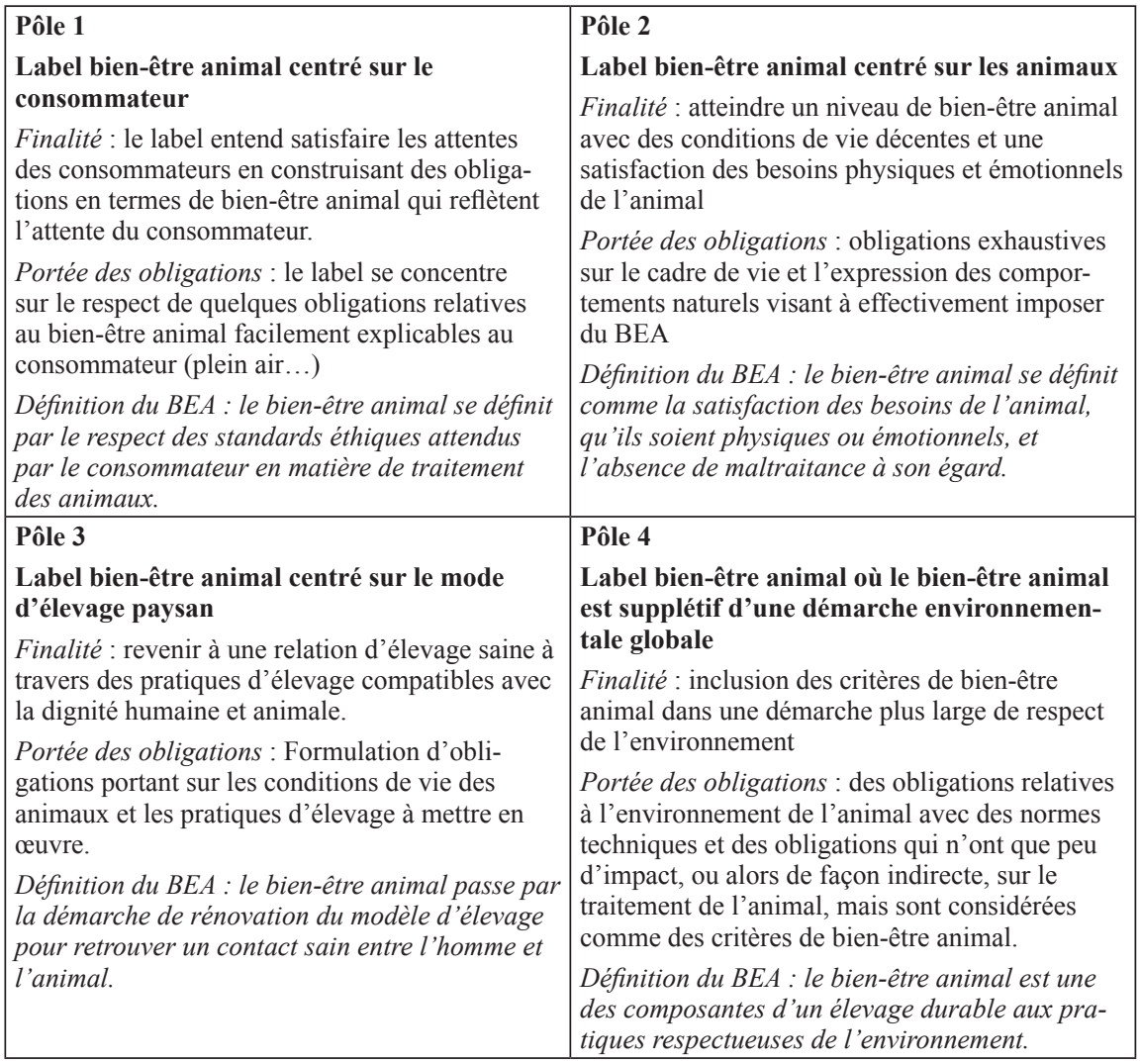

En prenant l'exemple de la production porcine, quatre labels illustrent ces différentes définitions et la prise en compte du bien-être animal.

50. Travail d'analyse des cahiers des charges des labels effectué par les étudiants menant le projet clinique Labelliser le bien-être animal, disponible sur demande auprès des auteurs.

51. À savoir : Certified Humane (États-Unis), Global Animal Partnership (États-Unis), Beter Leven (Pays-Bas), Animal Welfare Approved (États-Unis), Freedom Foods (Australie), Für Mehr Tierschutz (Allemagne), Naturafarm (Suisse), Nature \& Progrès (France et Belgique).

52. À savoir : le Label Rouge et le Label bio.

53. Cet article considère comme étant un label portant sur le bien-être animal tout label associé à un cahier des charges comportant des prescriptions relatives aux conditions de vie de l'animal, ou visant à améliorer son bien-être. 
- Pôle 1 : Définition du bien-être animal selon les attentes du consommateur: Global Animal Partnership

Le label américain Global Animal Partnership (GAP) ${ }^{54}$, créé à l'initiative de la chaîne de supermarchés Whole Foods Markets ${ }^{55}$, a pour ambition l'étiquetage de la sélection de produits la plus large possible, référencés en six niveaux. Plus le niveau est élevé, plus les exigences en matière de bien-être animal sont grandes. Tout comme Beter Leven, GAP promeut une démarche de progrès continu auprès des producteurs. Les six niveaux ${ }^{56}$ du label sont identifiables par le consommateur par l'usage d'un code couleur sur les produits renvoyant à des pratiques et obligations en matière de bien-être animal. Le label se concentre sur un nombre limité d'obligations en matière de bien-être animal que le consommateur pourra comprendre. Des critères symboliques et dont l'application par le producteur est facilement restituable en termes de communication commerciale auprès de l'acheteur sont privilégiés. Ainsi, chaque niveau rend compte du respect d'une obligation clé (par exemple : plein air, sans cage) sur la thématique de la liberté de mouvement de l'animal :

\begin{tabular}{|l|l|}
\hline Étape 1 & $\begin{array}{l}\text { Absence de cage, densité de peuplement limitée (pas de } \\
\text { surpeuplement). }\end{array}$ \\
\hline $\begin{array}{l}\text { Étape } 2 \text { « environnement } \\
\text { enrichi » }\end{array}$ & Les porcs doivent avoir accès à un environnement intérieur enrichi. \\
\hline Étape 3 « accès à l'extérieur » & Les porcs ont accès à l'extérieur. \\
\hline Étape 4 « élevage en plein air » & $\begin{array}{l}\text { Les porcs vivent en plein air de manière continue. Durant l'hiver, } \\
\text { les porcs peuvent être mis à l'abri, mais doivent bénéficier d'un } \\
\text { accès continu au plein air. }\end{array}$ \\
\hline $\begin{array}{l}\text { Étape } 5 \text { « absence de } \\
\text { mutilation » }\end{array}$ & $\begin{array}{l}\text { Les porcs sont élevés en plein air durant toute l'année et ne peuvent } \\
\text { être enfermés qu'en cas de conditions climatiques extrêmes. }\end{array}$ \\
\hline $\begin{array}{l}\text { Étape } 5+\text { «sur la même } \\
\text { ferme » }\end{array}$ & $\begin{array}{l}\text { Idem + les porcs doivent avoir vécu toute leur vie dans la même } \\
\text { exploitation. }\end{array}$ \\
\hline
\end{tabular}

- Pôle 2: Les besoins de l'animal au fondement de la définition du bien-être animal : Animal Welfare Approved (AWA)

Le label américain Animal Welfare Approved (AWA), créé par l'organisation non gouvernementale A Greener World ${ }^{57}$, a pour objectif de garantir aux animaux la possibilité de se comporter le plus naturellement possible dans les exploitations ${ }^{58}$. Pour ce faire, le label décline des catégories d'obligations qui entendent couvrir tous les aspects de la vie de l'animal d'élevage.

54. Voy. https://globalanimalpartnership.org/5-step-animal-welfare-rating-program.

55. Chaîne de distribution spécialisée dans les produits biologiques aux États-Unis.

56. 5-Step ${ }^{\circledR}$ Animal Welfare Rating Standards for Pigs, issued October 3, 2016, v2.2 (cahier des charges du label, p. 2).

57. https://agreenerworld.org/certifications/animal-welfare-approved/

58. « The premise of the Animal Welfare Approved standards is that animals must be allowed to behave naturally ». 
Les obligations qu'impose le label aux producteurs rendent compte d'une définition du bien-être animal fondé sur l'intégrité de l'animal. Les modalités de contrôle du respect des critères du label, notamment par le biais d'un audit organisé par l'ONG à l'origine du label, vont également dans le sens d'une telle définition. L'audit repose à la fois sur un suivi effectué par l'éleveur lui-même, chargé d'observer quotidiennement l'état de bien-être des animaux ${ }^{59}$, et sur le respect d'objectifs établis par l'éleveur ${ }^{60}$ en matière de bien-être animal qui lui imposent des pratiques préétablies.

Le contenu du cahier des charges suggère en outre un parti pris en faveur d'une définition large du bien-être animal. La section consacrée à l'espace de libre circulation des animaux ${ }^{61}$ entend ainsi favoriser un environnement d'élevage propice à l'expression des comportements naturels de l'animal par l'obligation d'accès au plein air ${ }^{62}$. Le label impose également aux producteurs d'intervenir, comme l'obligation de procurer des soins à tout animal malade ou blessé, de façon à immédiatement réduire la souffrance et la détresse ${ }^{63}$. Enfin, le cahier des charges du label comprend des obligations de ne pas faire, avec un principe d'interdiction des actes de maltraitance et de négligence envers les animaux ${ }^{64}$. L'ensemble des prescriptions du label AWA met en application une définition extensive du bien-être animal se fondant avant tout sur les besoins de l'animal.

- $\quad$ Pôle 3 : Définition du bien-être animal par le mode d'élevage paysan : Nature et Progrès

Le label Nature et Progrès résulte d'une démarche de l'association éponyme ${ }^{65}$ qui, à partir d'une charte, a établi une série de labels dédiés chacun à une espèce ${ }^{66}$. Ce label entend revaloriser les savoir-faire des éleveurs dans une optique de défense de l'agriculture paysanne et de l'élevage de taille raisonnée ${ }^{67}$. Ce mode d'élevage spécifique est considéré comme de nature à garantir le bien-être animal

59. Cahier des charges, article 4.0.2 : «All pigs must be thoroughly inspected at least twice per 24 hours $\gg$.

60. Ibid., article 3.0.4: «A health Plan emphasizing prevention of illness or injury must be prepared in consultation with the farm's qualified expert advisor to promote positive health and limit the need for treatment. "

61. «Ranging and foraging area access ».

62. Cahier des charges, article 7.0: «The aim of good ranging and foraging area management is to satisfy the herd's food-seeking behaviors. Animals must be able to explore the ground and their natural environment. 》

63. Ibid., article 3.1.1.

64. Ibid., article 12.0.1 : "Efforts must be made to develop positive relationships between the farmer and animals through gentle handling. "

65. https://www.ifoam.bio/en/nature-et-progres

66. http://www.natureetprogres.org/charte.pdf

67. « Les pratiques agricoles doivent permettre une santé optimale des animaux, des terres et de l'environnement alentour. Harmonieusement ancrée dans son territoire, la ferme joue un rôle social non négligeable : elle participe à l'économie locale et au maintien d'un tissu rural fortement menacé. » 
par la restauration d'une relation proche entre l'éleveur et ses animaux ${ }^{68}$. Aussi les cahiers des charges de ce label ${ }^{69}$ comprennent-ils des obligations tournées avant tout vers la promotion de pratiques d'élevage susceptibles de favoriser indirectement le bien-être animal, comme le localisme. Les cahiers des charges imposent la sélection d'espèces animales adaptées aux conditions climatiques et géographiques locales, en privilégiant les races de terroir faisant preuve d'une meilleure adaptation au milieu local du fait de leur rusticité ${ }^{70}$. Concernant la reproduction, les techniques d'insémination artificielle sont bannies ${ }^{71}$.

- Pôle 4 : Définition du bien-être animal par son inclusion dans une démarche environnementale plus globale : le label Agriculture biologique (AB)

Le label Agriculture biologique (AB) est, à l'origine, une norme publique régie par une réglementation européenne ${ }^{72}$. Cette réglementation impose des obligations générales qui entendent garantir au consommateur un modèle d'élevage respectueux de l'environnement. Si certaines de ces obligations sont relatives au bienêtre animal ${ }^{73}$, celui est défini avant tout comme une composante du respect de l'environnement. À ce titre, la réglementation européenne impose des obligations en matière de bien-être animal avant tout techniques et qui n'ont qu'un effet limité sur le bien-être animal. Ainsi, si la réglementation impose de manière détaillée des normes qui ont un effet bénéfique sur le bien-être animal, ces normes n'ont pas pour objectif premier l'amélioration du bien-être animal. Par exemple, la densité de terrain maximale à l'article 14(1)(b)(iv) du Règlement de 2007 dispose que « le nombre d'animaux est limité en vue de réduire au minimum le surpâturage, le tassement du sol $[\ldots] »$.

68. «Conscients que les éleveurs sont dépositaires d'une multitude de savoir-faire et de connaissances techniques, il est plus que jamais impérieux de promouvoir des systèmes d'élevage à visage humain reposant avant tout sur l'observation des animaux et le respect de leurs besoins physiologiques et éthologiques. »)

69. http://www.natureetprogres.org/servicepro/sp127.pdf, Introduction, p. 12.

70. http://www.natureetprogres.org/servicepro/sp127.pdf, Partie I réglementaire, article I.3.1 : «Le choix de la race que l'on souhaite élever est largement conditionné par le territoire dans lequel la ferme est inscrite (altitude, pluviométrie, type de sol et de végétation, relief...) et le type de conduite mis en place. Tout éleveur est incité à introduire dans son troupeau la ou les races les mieux adaptées aux conditions du milieu. Son choix se porte nécessairement sur des races locales ou des races à faible effectif, même si la ferme est située hors de leur berceau d'origine. L'éleveur met en place un plan d'action en ce sens. »

71. http://www.natureetprogres.org/servicepro/sp127.pdf, article 1.3.3 : « Nature \& Progrès encourage les éleveurs à s'affranchir des technologies liées à la reproduction et à se réapproprier la sélection de leurs animaux. »

72. Règlement $(\mathrm{CE}) \mathrm{n}^{\circ} 834 / 2007$ du Conseil du 28 juin 2007 relatif à la production biologique et à l'étiquetage des produits biologiques et Règlement (CE) n 889/2008 de la Commission du 5 septembre 2008 portant modalités d'application du règlement (CE) n ${ }^{\circ} 834 / 2008$ en ce qui concerne la production biologique, l'étiquetage et les contrôles, dont l'INAO assure l'application sur le marché français.

73. En particulier, les articles 11,12, 14, 15, 16, 17 et 18 du règlement $n^{\circ} 889 / 2008$. 


\subsection{La géométrie variable du bien-être animal dans la mise en ouvre du label : l'exemple de la concurrence entre les approches empiriques et sensorielles}

À chaque définition du bien-être animal correspond une méthode d'évaluation du niveau de bien-être éprouvé par les animaux. Les différentes approches de la mise en œuvre du bien-être animal au sein des labels mettent en relief la concurrence des différentes normes d'expertise : vétérinaires, zootechniciens et éleveurs ne sont pas régis par les mêmes impulsions ou les mêmes convictions quant à la mise en œuvre du bien-être animal. Il existe donc différentes approches de mise en œuvre d'une amélioration du bien-être animal selon les différents labels. Une première approche de la mise en œuvre du bien-être animal, qualifiée d' « approche empirique ", consiste à formuler des indicateurs du niveau de bien-être animal, collecter des données scientifiques sur la base de ces indicateurs et interpréter des résultats de manière scientifique (2.3.1). Une autre approche, qualifiée d' « approche sensorielle », se fonde sur l'observation visuelle de l'animal par des professionnels afin d'en déduire leur niveau de bien-être (2.3.2). Certaines méthodes d'évaluation, comme la grille d'évaluation Welfare Quality, associent approche empirique et approche sensorielle (2.3.3). En outre, des facteurs extérieurs à la problématique du bien-être animal, les contraintes économiques, interviennent dans le choix de la mise en œuvre des normes de bien-être animal.

\subsubsection{L'approche empirique}

Une approche empirique de la mise en œuvre du bien-être animal s'appuie sur des critères scientifiques, au premier rang desquels la mesure de la densité de l'élevage (soit le nombre d'animaux rapporté à la surface). Une forte densité étant le signe d'un faible niveau de bien-être animal, les labels fixent des seuils précis de densité à ne pas dépasser. Par exemple, le premier niveau du label Beter Leven garantit $2,25 \mathrm{~m}^{2}$ par truie, tandis que les niveaux 2 et 3 étoiles assurent $2,5 \mathrm{~m}^{2}$ par truie ${ }^{74}$. En plus du taux de densité des exploitations, l'approche empirique du bien-être animal se fonde sur des critères tels que l'analyse de la nature du sol, la mesure du taux d'humidité, la mesure des espaces de vie des animaux selon l'aménagement des stalles, l'évaluation de la condition physique de l'animal selon des critères vétérinaires - le rythme cardiaque de l'animal, par exemple.

La révolution numérique a contribué à l'émergence de l'utilisation de ce type d'indicateurs techniques pour mesurer à la fois l'environnement de l'animal et sa condition physique, donnant naissance à l' « élevage de précision ${ }^{75}$. L'élevage de précision peut être défini comme l'utilisation coordonnée d'outils technologiques,

74. Cahier des charges Beter Leven, https://beterleven.dierenbescherming.nl/fileupload/pdf_sterren_ consumenten/Tekst_BLk_varkens.pdf.

75. http://www.inra.fr/Grand-public/Dossiers/Les-agricultures-du-futur/L-elevage-de-demain-unelevage-de-precision/(key)/3 
notamment des capteurs, pour atteindre une efficacité maximale dans l'utilisation des ressources. Ces ressources incluent la main-d'œuvre, les ressources en alimentation animale, en eau, en espace, soit l'ensemble des coûts de production. Dans ce sens, l'approche empirique du bien-être animal correspond à la vision de l'élevage de précision, un élevage qui vise avant tout à améliorer le taux de productivité des exploitations animales, considérant le bien-être animal comme un facteur de productions parmi d'autres. Dans le cadre de l'élevage de précision, de nombreux dispositifs ont été conçus pour mieux détecter les températures anormales, maîtriser le contrôle de l'atmosphère des bâtiments et la distribution des aliments, ou encore l'organisation du temps de pâturage des animaux ${ }^{76}$. Un exemple d'une technologie de l'élevage de précision est celui des robots de traite faisant office de mini-laboratoires : équipées de capteurs, les machines évaluent l'état de santé de la vache lors de la traite en prenant la mesure de son rythme cardiaque et de sa température corporelle ${ }^{77}$. Le métier d'éleveur est appelé à évoluer en raison de l'approche empirique du bien-être animal telle qu'adoptée par l'agriculture de précision. Ainsi, au contact de ses bêtes, l'éleveur de précision se mue en manager chargé d'évaluer et d'interpréter les données générées par les outils technologiques, afin d'anticiper les difficultés et d'intervenir plus efficacement pour garantir aux animaux un niveau préétabli de bien-être animal.

La mise en place des outils de précision, garantissant l'application des critères de bien-être animal propres à l'approche empirique, vise à favoriser des exploitations productivistes par une rationalisation des facteurs de production tout en répondant à une demande sociétale de mieux en mieux informée sur les conditions de vie des animaux de ferme et donc plus exigeante en matière de bien-être animal. Par exemple, le LIT ${ }^{78}$ (Laboratoire d'innovation territorial) « Ouest Territoires d'Élevage » a pour objectif premier d'augmenter les volumes de production et de partir à la reconquête des parts de marché perdues au cours des dernières années (y compris des marchés à l'exportation) en développant des exploitations qui correspondent mieux aux attentes des consommateurs en matière de bien-être animal ${ }^{79}$.

\subsubsection{L'approche sensorielle s'appuie sur la relation particulière entre hommes et bêtes}

Comparée à l'approche empirique, l'approche dite « sensorielle » du bien-être animal s'appuie davantage sur la particularité de la relation multimillénaire existant entre Homme et bête dans le cadre de l'élevage. Plutôt que d'avoir recours à des

76. P. Mollier, « Ce que l’élevage de précision peut apporter au bien-être animal», INRA Science \& Impact, 2017, http://www.inra.fr/Chercheurs-etudiants/Systemes-agricoles/Tous-les-dossiers/ Bien-etre-des-animaux-d-elevage-la-recherche-pour-eclairer-le-debat/Ce-que-l-elevage-deprecision-peut-apporter-au-bien-etre-animal/(key)/4.

77. Ibid.

78. http://www.agro-media.fr/actualite/premiere-pierre-laboratoire-dinnovation-territorial-ouestterritoires-delevage-23731.html.

79. Site internet du LIT Ouest Territoires d'Élevage: https://www6.inra.fr/lit-ouest-territoireselevage. 
outils technologiques de pointe, cette approche se fonde sur les connaissances et le savoir-faire hérité de la pratique ancienne de l'élevage afin de déduire du comportement des animaux leurs besoins. Telle est l'approche du label Nature et Progrès, qui vise à promouvoir des systèmes d'élevage de taille petite à moyenne au sein desquels la mise en œuvre du bien-être animal repose avant tout sur l'observation des animaux et le respect de leurs besoins physiologiques et éthologiques ${ }^{80}$. Plutôt que de se fier à des données scientifiques collectées par l'intermédiaire de la technique, les éleveurs sont tenus de fonder leurs décisions sur une observation générale des animaux dans leur milieu de vie pour établir si ces derniers se trouvent dans un état de bien-être. Par exemple, une vache en état de bien-être aura tendance à se coucher naturellement d'une certaine manière. A contrario, un animal, qui ne parvient pas à se coucher, est susceptible de souffrir d'une pathologie. Cette approche, principalement basée sur l'observation - parfois longue - des animaux par des yeux expérimentés, valorise le savoir-faire de l'éleveur. Compte tenu des contraintes de temps et du niveau de formation des éleveurs à ces pratiques, cette approche est par nature incompatible avec l'élevage intensif.

En plus de promouvoir des exploitations de taille petite et moyenne, le label Nature et Progrès requiert que les exploitants soient indépendants, c'est-à-dire que les éleveurs ne soient pas liés contractuellement et exclusivement à un producteur ${ }^{81}$, afin de garantir que l'éleveur reste maître du volume de production et donc des conditions d'élevage de ses bêtes. Ainsi, le cahier des charges du label Nature et Progrès mentionne que le choix du bâtiment de logement est fait par l'éleveur en fonction des caractéristiques du terrain et des particularités physiologiques des animaux et des besoins comportementaux propres à leur espèce. Les besoins individuels de chaque animal, en fonction de son espèce, mais également de sa race, sont particulièrement pris en compte dans l'approche sensorielle, favorisant un suivi personnel de l'animal. De même, les mutilations sont interdites dans le cahier des charges de Nature et Progrès, à l'exception de la castration, autorisée dans certains cas seulement, et toujours en conformité avec la réglementation européenne stipulant que la castration ne doit pas être automatique, mais seulement utilisée en dernier recours. En cas de nécessité de procéder à la castration sur un animal, la procédure doit être effectuée sous anesthésie et l'animal doit ensuite bénéficier de soins de manière individualisée pour accompagner la guérison ${ }^{82}$. La prévention des maladies est par ailleurs privilégiée aux traitements curatifs.

En raison des difficultés tenant à l'évaluation de l'approche sensorielle du bien-être animal par un tiers, les cahiers des charges prévoient un système d'autoévaluation par lequel l'éleveur peut lui-même observer ses pratiques d'élevage de manière critique afin de les améliorer. En outre, une initiative européenne a vu

80. Travail d'analyse des cahiers des charges des labels effectués par les étudiants menant le projet clinique Labelliser le bien-être animal.

81. Ibid.

82. Ibid. 
une équipe d'experts, composée notamment de vétérinaires, mettre au point une méthode d'évaluation complète du bien-être animal, s'appuyant à la fois sur des critères scientifiques précis et sur l'observation des troupeaux par des yeux experts. Cependant, les travaux de cette initiative ont démontré qu'il demeure complexe pour des évaluateurs de pouvoir rapidement rendre un jugement sur la qualité des pratiques par la simple observation d'une exploitation en un temps limité et sans pouvoir se référer à des critères scientifiques précis.

\subsubsection{Une approche synergique du bien-être animal : l'exemple de Welfare Quality}

Le projet européen Welfare Quality a développé des processus standardisés d'évaluation du bien-être animal qui se focalisent sur l'animal lui-même : la condition physique, les blessures, mais également le comportement, tout en intégrant des critères managériaux comme la taille des enclos ${ }^{83}$. Les experts de 44 instituts de recherche et de 20 universités européennes ayant participé au projet sont partis du principe que fonder les mesures d'évaluation et de contrôle sur l'observation de l'animal permettait de mettre en exergue le résultat de son interaction avec son environnement ${ }^{84}$. Une telle méthode nécessite qu'une équipe d'experts se rende sur le lieu de l'élevage, y observe le troupeau et les animaux pendant un certain nombre d'heures, en vue d'évaluer leur état de bien-être selon le protocole précis préalablement établi. Toutefois, le travail de terrain a révélé que les protocoles établis, très complets, étaient trop lourds et trop coûteux pour pouvoir être généralisés. Pour plus de praticité, les protocoles Welfare Quality ont donc été déclinés en protocoles partiels dans certains élevages. 46 projets - déjà mis en place ou prévus - basés sur le projet Welfare Quality sont actuellement recensés ${ }^{85}$.

Les différentes approches de mise en œuvre du bien-être animal au sein des élevages se croisent au sein des cahiers des charges des labels dédiés, proposant aux individus désireux d'améliorer leurs pratiques en matière de bien-être animal de nombreuses pistes de mise en œuvre. Si ces différentes modalités d'intégration du bien-être animal dans les pratiques ne se font pas concurrence entre elles, elles correspondent bien à des modes de production en forte concurrence : l'élevage et les productions animales. Toutefois, la très complète méthode d'évaluation Welfare Quality, qui combine les deux approches, empirique et sensorielle, laisse entrevoir la possibilité d'une mise en œuvre équilibrée du bien-être animal dans le cadre de la labellisation.

83. Site internet de Welfare Quality Network : http://www.welfarequality.net/en-us/home/.

84. Ibid.

85. Ibid. 


\section{CONCLUSION}

Les différents labels qui éclosent progressivement dans le domaine du bien-être animal reproduisent les divergences existantes entre différents systèmes normatifs en concurrence pour définir le bien-être animal et les modalités de son évaluation. Tandis que la normativité publique tendrait à trancher en faveur d'une démarche scientifique du bien-être animal particulière, les normativités privées exploitent au contraire une diversité d'approches sur la question du bien-être animal. À ce titre, la labellisation privée a pu renforcer la créativité scientifique en matière de bienêtre animal. Il y a lieu d'espérer que les acteurs privés tirent les enseignements des différentes approches poursuivies afin d'affiner la définition du bien-être animal et sa mise en œuvre dans les labels. De cette analyse pourrait déboucher une hybridation des approches du bien-être animal, à même de prendre pleinement en compte le caractère multifactoriel de cette problématique.

Si une telle prolifération d'initiatives de labellisation du bien-être animal a pour vertu de permettre de vérifier sur le terrain quelles démarches scientifiques rendent possible une amélioration réelle du bien-être des animaux, ce foisonnement d'initiatives en matière de bien-être animal a pour pendant le risque de confusion du consommateur. En effet, si les approches du bien-être animal de chaque label sont souvent complémentaires et peuvent répondre aux attentes particulières de chaque consommateur, celui-ci n'est souvent pas suffisamment à même de les appréhender. Il convient donc d'améliorer la clarté, la précision et la fiabilité des informations fournies sur les approches du bien-être animal poursuivies par chaque label. La délivrance d'informations adéquates renforcerait la compréhension de la question par le consommateur et sa capacité à adapter ses choix de consommation à sa sensibilité pour le bien-être animal. Peut-être plus important encore, une information adéquate serait utile également au producteur, une fois tenu d'une obligation de produire des informations qu'il n'était pas accoutumé à prendre en compte, afin qu'il modifie ses pratiques pour mieux répondre à une demande croissante des citoyens pour des produits respectueux des animaux. Auquel cas, la concurrence des normativités en matière de bien-être animal pourrait continuer à renforcer la prise en compte du bien-être animal par le consommateur dans ses décisions d'achat et par le producteur dans ses pratiques. L'avenir dira si in fine cette information contribue à son tour à l'amélioration des conditions de vie des animaux d'élevage.

Au niveau juridique, le foisonnement des initiatives en matière de bien-être animal peut en outre faire douter de voir un jour émerger un référentiel unitaire. Ainsi, ce phénomène de concurrence entre les normes participe d'une crise de la normativité juridique : lorsque la loi refuse de trancher dans l'indétermination du réel, le législateur préfère laisser les acteurs du marché choisir la norme qui deviendra la référence de facto. L'intervention du législateur peut néanmoins ressurgir au gré de l'élaboration de la norme, que ce soit simplement pour sanctuariser la norme 
désignée comme préférable par le marché, mais éventuellement aussi pour corriger le marché de façon à le pousser à choisir des normes de meilleure facture. Le législateur intervient alors de manière indirecte par le biais de la commande publique ou plus directement via la réglementation.

\section{SUMMARY: COMPETING STANDARDS IN ANIMAL WELFARE LABELLING}

For the last decade, the animal agriculture industry in France has suffered from rising levels of consumer distrust. The debate around animal agriculture has shifted away from controversies on food safety and traceability, such as the notorious Mad Cow Crisis in the late 1980s or the more recent 2013 horsemeat scandal, to questioning the very existence of raising animals for food. Today in France, as in any other nations where animal agriculture has undergone intensification, exposés by animal protection groups regularly make the front page of newspapers, thereby revealing the inhumane, yet common, industry practices of intensive livestock production to the general public.

Criticism directed towards industrial farm animal production, although solely aimed at intensive livestock production, tends to address animal agriculture as a whole, thus further accelerating the demise of small, independent, and more humane farming. To avoid confusion in the eye of the public, producers and animal protection advocates have identified consumer information as a potentially powerful tool to allow more humane producers to better signal themselves on the market and to improve animal welfare practices in animal agriculture. However, when left unregulated, consumer information demonstrably reinforces confusion among consumers rather than influencing consumption patterns (and production methods in turn). Such is the case in France, where the proliferation of labeling initiatives in the private sector has fostered confusion around animal welfare-related consumer information. Along with this proliferation in labels has come lacking, inadequate, and opaque consumer information, which may lead to consumer distrust while creating significant obstacles with regard to the assessment of the effectiveness of good consumer information.

Defining good quality consumer information on farm animal welfare is necessary to avoid consumer distrust and to allow for the assessment of the effectiveness of good consumer information on the improvement of animal welfare practices in animal agriculture. This article therefore attempts to define farm animal welfare through an analysis of the different farm animal welfare standards of labels in France, the European Union, the Netherlands, and the United States. These standards vary widely from one label to another, thus revealing an intensely competitive environment in terms of the definition of what constitutes a good farm animal welfare standard; how and by whom a farm animal welfare standard should be implemented; and how to assess the reliability of such a standard. 
This article focuses on two main areas where standards compete. The first relates to the person enacting the standards: a public or a private entity. The second involves the definition of animal welfare undermining a standard.

The discussion on who should enact and implement a standard leads to a classical discussion on the competition between the public and the private sectors as normative bodies. Administrations have often voiced their concerns over the proliferation of private norms through labeling on the marketing of goods, which they see as a risk to the good functioning of the marketplace. Yet, public authorities have been quite reluctant to enact standards themselves, thus leaving room for many private initiatives. Private and public entities can work together to ensure good consumer information: one label in the Netherlands, Beter Leven (literally: "better life") provides a good example of the complementarity of public and private bodies in the enactment and implementation of a farm animal welfare standard. Another example of a fructuous dialog between the private and public sectors is the one that led to the law on the mandatory labeling of shell eggs in the EU.

In a second part, this article explores the competition between different definitions of farm animal welfare. The proliferation in the number of farm animal welfare-related labels reveals an intense competition taking place at an earlier stage in the conception of a label, when defining the concept of what is, or should be, farm animal welfare. Each label has its own definition of farm animal welfare, with each depending on different disciplines and knowledge: biology, veterinary science, ethology, old animal husbandry skills, etc. These different definitions also compete with each other. This section identifies one central division in the field of animal welfare, between animal welfare as defined by animal husbandry and animal science. This division in the theory of farm animal welfare is reflected further down in the process of defining animal welfare standards in a given label, and at the time of implementing, monitoring, and assessing these standards.

In conclusion, the intense competition taking place between all the different actors responsible for the creation, implementation, and regulation of labels on farm animal welfare is reflective of a broader crisis of regulation affecting the power of the law. As public authorities are increasingly more reluctant to enact standards on production methods, the private sector and nonprofits are more likely to step in, thus further eroding the unity and consistency of norms. However, one should not take the absence of the public sector for granted. Far from giving up on its prerogative, the state still occasionally intervenes on markets to regulate and harmonize standards. In the case of farm animal welfare standards, such an intervention takes the form of rules on public procurement.

Mots clés : bien-être animal, labellisation, concurrence, information du consommateur, normativités

Keywords: animal welfare, labeling, competition, consumer information, norms 